\title{
MIRAO: a mid-IR adaptive optics system design for TMT
}

\section{Mark R. Chun, Jay Elias, Brent Ellerbroek, Tim Bond, Ming Liang, et al.}

Mark R. Chun, Jay Elias, Brent Ellerbroek, Tim Bond, Ming Liang, Richard Clare, Alan Tokunaga, Matt Richter, Larry Daggert, "MIRAO: a mid-IR adaptive optics system design for TMT," Proc. SPIE 6272, Advances in Adaptive Optics II, 62720 S (27 June 2006); doi: 10.1117/12.670062

Event: SPIE Astronomical Telescopes + Instrumentation, 2006, Orlando, Florida, United States 


\title{
MIRAO: A Mid-IR adaptive optics system design for TMT
}

\author{
Mark R. Chun*a, Jay Elias ${ }^{\mathrm{b}}$, Brent Ellerbroek $^{\mathrm{c}}$, Tim Bond ${ }^{\mathrm{a}}$, Ming Liang ${ }^{\mathrm{b}}$, Richard Clare ${ }^{\mathrm{c}}$, Alan Tokunaga ${ }^{\mathrm{a}}$, \\ Matt Richter ${ }^{\mathrm{d}}$, Larry Daggert ${ }^{\mathrm{b}}$ \\ anstitute for Astronomy, University of Hawaii, 640 N. A'ohoku Place, Hilo, HI 96720; \\ ${ }^{b}$ National Optical Astronomy Observatory, 950 N. Cherry Avenue, Tucson, AZ, 85710; \\ ${ }^{\mathrm{c}}$ Celtco/TMT 1200 E. California Blvd, Pasadena, CA 91107; \\ ${ }^{\mathrm{d}}$ Department of Physics, University of California, One Shields Avenue, Davis, CA 95616-8677.
}

\begin{abstract}
We present a design of a thermal-infrared optimized adaptive optics system for the TMT 30-meter telescope. The approach makes use of an adaptive secondary but during an initial implementation contains a more conventional ambient-temperature optical relay and deformable mirror. The conventional optical relay is used without sacrificing the thermal background by using multiple off-axis laser guide stars to avoid a warm dichroic in the common path. Three laser guide stars, equally spaced 75" off axis, and a "conventional" 30x30 deformable mirror provide a Strehl > 0.9 at wavelengths longer than 10 microns and the LGS beams can be passed to the LGS wavefront sensors with pickoff mirrors while a one-arcminute field is passed unvignetted to the science instrument and NGS WFSs. The overall design is relatively simple with a wavefront correction similar to existing high-order systems (e.g. 30x30) but still provides competitive performance over the higher-order TMT NIR AO design at wavelengths as short as 3 microns due to its reduced thermal emissivity. We present our figures of merit and design considerations within the context of the science drivers for high-spectral resolution NIR/MIR spectroscopy at 5-28 microns on a 30-meter ground-based telescope.
\end{abstract}

Keywords: adaptive optics, mid IR, thermal emissivity, laser guide stars, large telescopes

\section{INTRODUCTION}

At mid-infrared wavelengths ground-based observations are background-limited and the observing time to reach a fixed signal-to-noise ratio on point sources for a telescope at the diffraction limit decreases with the fourth power of the telescope diameter. At these wavelengths, the 30-meter TMT telescope can provide an increase in speed of 2 to 4 orders of magnitude over current ground-based capabilities permitting study of a variety of astronomical phenomena such as star and planet formation at unprecedented sensitivities at high spectral and spatial resolution (see Elias et al. 2006).

The principal source of background radiation for observations made at mid-infrared wavelengths from the ground is the thermal emission from ambient temperature structures (e.g. atmosphere, telescope, enclosure, and instrument). This thermal emission becomes a significant source of background noise for wavelengths longer than 2 microns but at 5-28 microns this thermal background is the dominant noise source for all but the brightest handful of objects in the sky.

Provided that the background can be subtracted to the limit of photon statistics, the only way to increase sensitivity is by increasing telescope aperture. However, with the increase in telescope aperture size comes the additional burden of correcting for the incoherence of the atmospherically distorted wavefront across the aperture in order to continue work at the diffraction limit. For the current class of 4-8 meter telescopes, the phase variance across the telescope aperture is typically less than 1 radians $^{2}$ at the longer wavelengths so little or no adaptive correction is required. This will no longer be true for the next generation of large telescopes.

While long wavelength observations are near diffraction-limited, those interested in diffraction-limited observations at shorter mid-infrared wavelengths (e.g. 2-5 microns) have suffered from the greatly increased thermal background from a conventional ambient temperature adaptive optics systems or resorted to implementing an adaptive secondary mirror (Lloyd-Hart 2000) that affords an increase in the optical throughput in addition to the lower thermal emission. LloydHart (2000) shows that at the shorter thermal wavelengths (2-10 microns) a telescope equipped with an adaptive secondary provides gains in observing time over a conventional adaptive optics system of 3-4. Nearly all of this gain is due to the reduction of the thermal emission from the adaptive optics system. Another option for lowering the emissivity of an adaptive optics system is to cool the optical relay to cryogenic temperatures (Herriot et al. 2006). Work on

Advances in Adaptive Optics II, edited by Brent L. Ellerbroek, Domenico Bonaccini Calia,

Proc. of SPIE Vol. 6272, 62720S, (2006) - 0277-786X/06/\$15 - doi: 10.1117/12.670062

Proc. of SPIE Vol. 627262720 S-1 
deformable mirrors that operate at cryogenic temperatures is in progress though this development is driven in large part by space -based missions with somewhat disparate requirements (e.g. size and stroke).

With the advent of the next generation of large telescopes, an enormous leap in mid-infrared capabilities will be possible. To obtain the $\mathrm{D}^{4}$ gain, an adaptive optics system is needed but it must provide the corrected wavefront without severely increasing the thermal emissivity of the system. In this paper we present a design for a mid-infrared optimized adaptive optics system (MIRAO) for a mid-infrared echelle spectrograph (MIRES) on TMT that uses a novel technique to minimize the increase in system emissivity with a conventional adaptive optics relay.

\section{TMT MID-INFRARED ECHELLE SPECTROGRAPH}

\subsection{MIRES Science}

In regards to the design of the mid-IR adaptive optics system it is important to understand the types of observations and types of objects that drive the requirements of MIRES and MIRAO. The science case for MIRES/TMT is described elsewhere (Elias et al. 2006).

The versatility of a mid-IR echelle spectrograph in addressing astrophysical issues arises from the richness of the midinfrared region of the spectrum, which includes spectral features of atoms, ions and molecules. Hence, spectroscopy at these wavelengths can be used to probe gas in environments ranging from planetary atmospheres, to cool molecular clouds, HII regions, and active galactic nuclei. Mid-infrared molecular spectroscopy is particularly well suited to probing the physical conditions and chemistry in protostellar envelopes and the inner planet formation regions $(<5 \mathrm{AU})$ of disks. The mid-infrared also allows us to observe environments over a range of extinction, even those that are obscured by fifty or more magnitudes of visual extinction. Furthermore, emission from dust in a variety of astrophysical environments reaches a maximum at mid-infrared wavelengths. High-spatial, high-contrast imaging with MIRES will provide an exquisitely detailed look at the spatial structure of these regions.

\subsection{Requirements for MIRAO}

The driving requirements of the system are the science observation wavelengths ( $8-25$ microns) and the fact that the science objects themselves are heavily embedded in dust and small (unresolved to a few arcseconds in size). This requires a low-emissivity system (e.g. AM2, cryogenic DM) and wavefront sensors (WFS) that work with laser guide stars LGS(s) or natural-guide star (NGS) WFS working at NIR wavelengths. The core requirements as derived from the TMT Science Requirements Document are specified below in Table 1.

Table 1Core Science Requirements for MIRAO

\begin{tabular}{|l|l|}
\hline Science Wavelength Range & $2.8-28$ microns. \\
\hline FOV & Small FOV science instrument: 10" with 1 arcmin goal \\
\hline Wavefront Quality & Requirement wavefront rms phase $<750 \mathrm{~nm}$, goal $<350 \mathrm{~nm}$ rms \\
\hline Photometry & "All sky" and limited only by the natural tip-tilt stars. \\
\hline Astrometry & Systematic, uncalibrated errors $<5 \%$ in the N band over 1 arcmin field. \\
\hline Background & $\begin{array}{l}\text { Differential astrometry is important, and the AO system should provide sufficient calibration } \\
\text { information to not degrade the astrometric capabilities beyond the limits set by the atmosphere. }\end{array}$ \\
\hline $\begin{array}{l}\text { Instrument should not increase N-band background by more than } 15 \% \text { over nature sky+telescope } \\
\text { background (assumed to be } 5 \% \text { ). This results in an absolute emissivity of less than } 1 \% \text { for the } \\
\text { combination of MIRES/MIRAO. }\end{array}$ \\
\hline
\end{tabular}

Technically, we identify the highest risk potential components of MIRAO as the adaptive secondary (AM2), cryogenic deformable mirrors, a chopping secondary mirror, and the advent of high-speed low-noise near-infrared detectors for natural guide star wavefront sensors. These drove our design of the system. 


\section{AO PERFORMANCE SIMULATIONS}

As input to the first-order design of the AOS, we ran simulations of a number of system configurations. These included analytic as well as Monte Carlo simulations. In general the simulations provided comparable results though for the initial specifications of the system, we considered both the simulation results and more practical considerations such as the benefits of duplicating design choices made for the facility near-IR adaptive optics system (NFIRAOS, references to papers during this conference).

The site conditions have a strong effect on the performance of the system. However, since the TMT site is not chosen, we have assumed that the site conditions were for a mid-Chilean site with median seeing of 0.67 " seeing at 0.5 microns.

\subsection{Error Budget}

Table 2: Top-level summary of the MIRAO error budget

\begin{tabular}{|c|c|c|c|}
\hline \multicolumn{4}{|l|}{ MIRAO Error Budget } \\
\hline v 2.5.1 25 Jan 2006 & Best & Worst & Comments \\
\hline \multicolumn{4}{|l|}{ AO High order errors } \\
\hline Tomography + DM fitting & 310.0 & 310.0 & 3 bright LGS in a 70" radius asterism, median CP seeing \\
\hline WFS & 35.0 & 39.0 & LGS WFS Noise \\
\hline $\mathrm{DM}$ & 25.0 & 25.0 & Hysteresis: LG's modeling 29 May 05 \\
\hline LGS & 0.0 & 100.0 & Na Layer Tracking (Guess) \\
\hline Control Servo & 27.0 & 28.3 & Servo Lag, RTC round-off \\
\hline $\begin{array}{l}\text { Residual MIRAO optics errors (after AO } \\
\text { correction) }\end{array}$ & 57.0 & 64.0 & $\begin{array}{l}\text { Common path errors equal to the non-common path errors } \\
\text { but with } 75 \% \text { corrected by MIRAO. }\end{array}$ \\
\hline \multicolumn{4}{|l|}{ Telescope } \\
\hline Residual telescope aberrations & 50.0 & 88.0 & $\begin{array}{l}\text { From RFP. Scaling to "baseline" done by LJ with PAOLA } \\
15 \text { Aug 05, this is based on NFIRAOS correction (!) and a } \\
\text { guess for the telescope aberrations }\end{array}$ \\
\hline Obscuration from M2 support structure & 30.0 & 30.0 & Initial simulation by LG 15 Aug 05 \\
\hline Telescope Coma (finite range to LGS) & 0.0 & 35.0 & $\begin{array}{l}\text { Worst case is based on tilting lens mechanism that } \\
\text { accounts for LGS range. Best case assumes offsets can be } \\
\text { put into WFS to make a further reduction. }\end{array}$ \\
\hline \multicolumn{4}{|r|}{ P } \\
\hline Residual science instrument aberrations & 50.0 & 160.0 & $\begin{array}{l}\text { Best: } 50 \mathrm{~nm} \text { rms, Worst: } 99 \% \text { Strehl at } 10 u m \text { to the slit of } \\
\text { the spectrograph. Includes rotator, non-common path } \\
\text { between WFSs in dewar, and static optic errors. }\end{array}$ \\
\hline \multicolumn{4}{|l|}{ Tip-tilt errors } \\
\hline $\begin{array}{l}\text { Residual atm/telescope windshake (TT) } \\
\mathrm{nm} \text { rms }\end{array}$ & 140.0 & 400.0 & $\begin{array}{l}\text { RC } 2 \text { Sept } 05 \text {, worst equals } 95 \% \text { sky coverage at } \mathrm{l}=30 \text { and } \\
\text { is } 400 \mathrm{~nm} \text { wavefront error, Assumes } \mathrm{r} 0=0.15 \mathrm{~m} \text { and } \\
\mathrm{L} 0=30 \mathrm{~m} \text {, sampling rate } 400 \mathrm{~Hz} \text {, Baseline } 20 \mathrm{~Hz} \text { TT } \\
\text { platform, Note NFIRAOS does better by planning to use } \\
\text { DM for high-speed TT correction (split bandwidth } \\
\text { DM/TTM). Note that this error includes windshake, NGS } \\
\text { WFS noise, tilt anisoplanatism, and servo lag terms }\end{array}$ \\
\hline mas rms & 3.9 & 11.0 & \\
\hline Delivered wave-front error & 360 & 560 & (nm rms) \\
\hline Strehl@3.3um & 0.59 & 0.27 & \\
\hline Strehl@4.6um & 0.76 & 0.48 & \\
\hline Strehl@10um & 0.94 & 0.84 & \\
\hline Strehl@20um & 0.99 & 0.96 & \\
\hline
\end{tabular}


For the feasibility design of MIRAO we constructed a basic error budget for the residual wavefront error from the residual atmospheric aberrations to the entrance slit of the MIRES spectrograph. The error budget (Table 2 below) is intended to provide an accounting of all the major contributing errors in the residual optical wavefront up to the slit of the spectrograph. The budget during the feasibility study is intended to identify the most significant terms and understand how they drive the design of the instrument. Details within the error budget will be added throughout the design process.

The MIRAO/MIRES error budget follows an early NFIRAOS budget by separating the higher-order errors and the tip/tilt residual errors. Higher-order errors are combined in a root-sum-square (RSS) and a high-order Strehl is derived using the Marechal approximation. The jitter errors are converted to rms angle of arrival errors, combined (RSS), then converted to a Strehl degradation due to residual jitter via the following formalization:

$$
\text { Streh } \left._{\text {tilt }} \sim 1 /\left[1+\pi^{2} / 2 *\left(\sigma_{\theta, \text { tilt }} \text { [radians }\right] /(\lambda / D)\right)^{2}\right]
$$

Finally, the final delivered Strehl is taken as the product of the higher-order and jitter Strehls. It is important to recognize that these two errors affect the image profile in different ways. The high order errors mainly remove light from the diffraction core, while the jitter errors act to broaden it. The light in the broadened core can in principle be recovered, at the expense of increased background, using a wider slit or photometric aperture.

A top-level summary of the error budget is given in Table 2 above. The major errors contributing to this budget are the WFS tomography and DM fitting errors, the residual tip/tilt jitter error, and the static optical non-common path errors. The static optical non-common path errors are currently a placeholder. During conceptual design these will be detailed and replaced by the detailed component budget. The residual jitter errors are principally an issue of sky coverage. These calculations are discussed in Section 3.3 - Sky Coverage Simulations: Tip/Tilt/Focus WFS. The tomography and DM fitting calculations made using a variety of simulation packages. These simulations are the subject of the next section.

\subsection{High-order Simulations}

The initial goals of the AO simulations were to define the basic specifications for MIRAO to meet the science requirements set out by the science case and the SRD and provide first-order performance and image quality numbers to feed back to the team developing the science case. In all cases a standard Cerro Pachón turbulence profile with an $\mathrm{r} 0=15 \mathrm{~cm}$ at $0.5 \mathrm{um}$ and $\mathrm{L} 0=30 \mathrm{~m}$ was used, the LGS were assumed to be bright (equivalent to a $\mathrm{V}=6^{\text {th }}$ magnitude star), and the projection of the LGS was from behind the secondary mirror.

The first simulations were made using a purely analytic simulation package (PAOLA). This package was limited to seeing-limited, natural-guide star (NGS), and single laser guide star (LGS) configurations. These simulations confirmed that the order of correction required for MIRAO is modest in comparison to the facility AOS and that a WFS of order 30x30 would provide excellent performance at MIR wavelengths and modest image quality in the NIR. An AOS containing this number of degrees of freedom is comparable to existing AOSs (e.g. Starfire, Gemini MCAO), can be implemented with existing WFS and DM technologies, and is viewed as a relatively low-risk implementation. We take 30x30 subapertures as our default WFS configuration.

Table 3 Strehl Results from High-order simulations

\begin{tabular}{|l|c|c|c|c|}
\hline Configuration & 3.3micron & $\mathbf{4 . 6 m i c r o n}$ & 10micron & 20micron \\
\hline NGS, N_DM=15, J 11 & 0.44 & 0.62 & 0.91 & 0.98 \\
\hline NGS, N_DM=15, J 12 & 0.13 & 0.25 & 0.76 & 0.94 \\
\hline 1LGS, N_DM=15, on-axis & 0.27 & 0.51 & 0.88 & 0.95 \\
\hline 1LGS, N_DM=30, on-axis & 0.57 & 0.75 & 0.96 & 0.98 \\
\hline 3LGS, N_DM=15 & 0.60 & 0.77 & 0.95 & 0.98 \\
\hline 3LGS, N_DM=30 & 0.78 & 0.88 & 0.97 & 0.99 \\
\hline
\end{tabular}

For the more detailed simulations, three configurations were considered. First, the NGS AO cases with $15 \times 15$ and $30 \times 30$ WFSs bracket the performance of the system. For the case of MIRES, some of the science targets will be bright enough to use as NGSs in the NIR. Our baseline for MIRAO is to support the use of a NGS for AO WFS either in the J-band or H-band provided a suitable NIR detector is available. Two LGS AO cases were simulated. A single LGS was 
considered both on-axis and off-axis and a three-LGS system with all LGSs off-axis was considered. The off-axis LGS cases were considered in support of a pierced/pickoff mirror to feed the LGS WFSs with a zero emissivity impact on the science NIR/MIR beams. In both cases the LGS off-axis distance is 70-75 arcseconds and is set by the desire to feed a full 60 arcsecond diameter field of view to the MIRES spectrograph and its NGS WFSs; this field of view is driven primarily by the need for adequate sky coverage (see section 3.3). We note that the off-axis 1LGS case suffers from significant anisoplanatism and focal-anisoplanatism.

\subsection{Sky Coverage Simulations: Tip/Tilt/Focus WFS}

The second set of simulations run during the study considered the required field of view of the NGS Tip/Tilt/Focus WFS to obtain a given level of residual jitter and focus error. Richard Clare (TMT) ran these simulations for the case of star fields at the galactic pole as well as at galactic latitude of 30 degrees and galactic longitude of 180 degrees. The latter case was taken as our baseline scenario since most of the science targets for MIRES are stellar and in or near the plane of the galaxy. The NGS TTF WFS is assumed to be running in the NIR and for these calculations J-band was used. Because the J-band star counts do not go sufficiently faint, we extended the Spagna star counts using a simple power law. The Spagna counts were extrapolated 2 magnitudes for the simulations. The figure below illustrates the probability of finding a NGS for Tip/Tilt/Focus in the J-band as a function of residual jitter error.

The important conclusion of these simulations are (1) that a $\varnothing 30$ " acquisition field for the TTF WFS is insufficient. There are simple not enough stars to provide a large sky coverage, and (2) there is no advantage to having an acquisition field larger than $\varnothing 90 "$ ". The requirement on residual jitter error for MIRAO/MIRES is somewhat relaxed since the slit throughput is relatively insensitive to jitter errors. For a slit width of $1.5 \lambda / \mathrm{D}$ at $10 \mathrm{microns}$, an rms jitter error of $11 \mathrm{mas}$ (400nm rms phase) has a negligible impact on the slit throughput and sensitivity of MIRAO/MIRES. This is the worstcase jitter error for a $\varnothing 60$ " acquisition field. From a practical standpoint, the NGS TTF WFS acquisition field of view drives the size of the MIRES dewar window since it resides inside the spectrograph dewar. A $\varnothing 60 "$ FOV translates into a large but available window size.

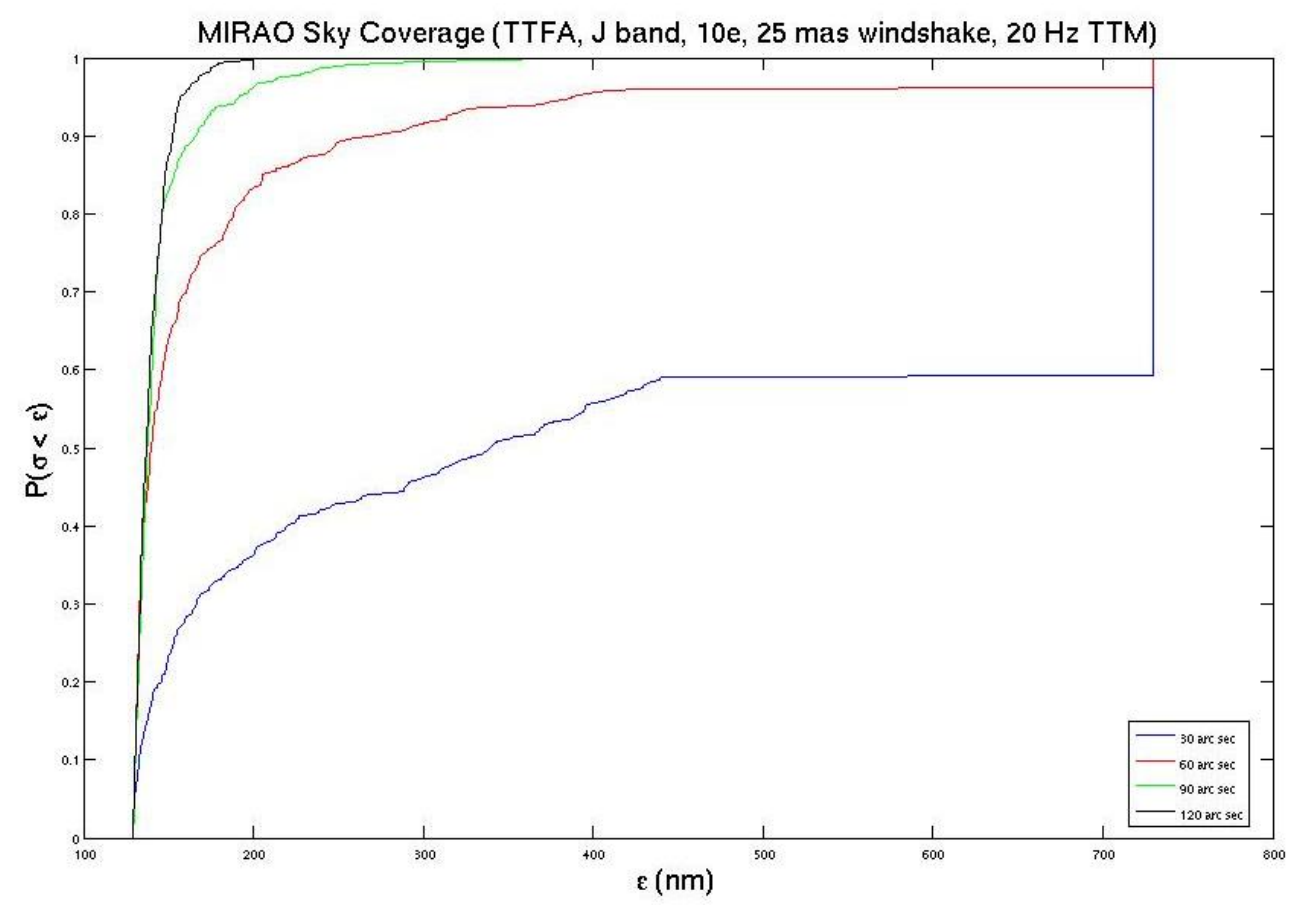

Figure 1- Sky coverage vs. NGS patrol field. The fractional coverage is shown as a function of desired maximum tip/tilt wavefront error in $\mathrm{nm}$. This simulation uses an extended Spagna model at galactic latitude of 30 degrees and galactic longitude of 180 degrees. The NGS acquisition fields considered for the TTF WFS were Ø30"(blue), Ø60"(red), $\varnothing 90 "$ (green), and $\varnothing 120 "$ (black). 


\subsection{Summary of final configuration performance}

The MIRAO Strayman consists of 3 off-axis LGSs (optional NGS AO), a single NGS TTF WFS with a 60" acquisition FOV, and 30x30 DM/AM2 correction. Its performance in context of the MIRAO SRD requirements is given in Table 4 below. In all cases the design can meet or exceed the SRD requirements. In the case of the emissivity, the design goal of $1 \%$ can only be met in the upgraded AM2 configuration.

Table 4. Summary of MIRAO Strawman Design Performance

\begin{tabular}{|l|c|c|c|}
\hline & SRD & MIRAO & \multicolumn{2}{|c|}{ MIRAO/AM2 } \\
\hline Delivered field of view & $10^{\prime}, 1$ ' goal & 1 & \multicolumn{2}{|c|}{$\sim 95 \%$} \\
\hline Sky coverage & limited by TTGS & \multicolumn{2}{|c|}{$350-550 \mathrm{~nm} \mathrm{rms}$} \\
\hline Residual Wavefront error & $<350 \mathrm{~nm}$ (goal) & \multicolumn{2}{|c|}{$>98 \%$} \\
\hline Optical throughput & $>85 \%$ & $>95 \%$ & $1 \%$ \\
\hline Thermal emissivity(10um) & $1 \%$ & $4 \%$ & \multicolumn{2}{|c|}{} \\
\hline
\end{tabular}

\section{MIRAO DESIGN}

\subsection{Overview}

During this design we adopted the philosophy that as a potential first generation instrument on TMT, the new science enabled by MIRAO/MIRES should be delivered by an instrument that focuses on the core science case and minimizes technical risks as much as possible; capabilities outside those needed for the core science should not increase the risk/complexity of the implementation.

The final configuration for MIRAO was the result of a trade study of several configurations including a 'cooled' AOS (T - 35C), an ambient temperature AOS with pickoff/pierced mirrors feeding the LGS WFSs, and an adaptive secondary configuration.

In order to compare the performance of the approaches, we used a simple metric for system performance based on the assumption that most of the science targets are point sources and that the observations are background limited. The metric is as follows and is a measure of the rate at which faint, background-limited point sources can be measured.

$$
\text { (throughput*Strehl })^{2} / \text { (system emissivity). }
$$

System emissivity includes the contributions from sky, telescope, MIRAO and MIRES. We consider only the throughput associated with the $\mathrm{AO}$ system in this metric, since the instrument throughput is presumably the same for the different AO configurations. As a result, the throughput of the AM2 configuration is $100 \%$.

Table 5: Representative Figure of Merit Values

\begin{tabular}{|l|c|c|c|c|}
\hline \multicolumn{1}{|c|}{ Configuration } & $\mathbf{3 . 3} \mathbf{u m}$ & $\mathbf{4 . 6 u m}$ & $\mathbf{1 0 u m}$ & $\mathbf{2 0 u m}$ \\
\hline Cool AOS, 3LGS, 15x15 DM & 0.18 & 0.27 & 0.38 & 0.37 \\
\hline Ambient AOS, pickoff mirrors, 3LGS, 15x15 DM & 0.22 & 0.36 & 0.59 & 0.62 \\
\hline Ambient AOS, pickoff mirrors, 3LGS, 30x30 DM & 0.37 & 0.48 & 0.61 & 0.64 \\
\hline Ambient AOS, pickoff mirrors, 3LGS, AM2 & 0.61 & 0.77 & 0.94 & 0.98 \\
\hline
\end{tabular}

Both the AM2 and cryogenic DM options are seen as technical challenges that may not be ready at the time of first-light of the telescope. While the adaptive secondary is a desirable correcting element for MIRAO given the low emissivity and the increased overall optical throughput, we decoupled MIRAO/MIRES from the delivery of AM2 by considering a two-phase implementation. First, the AOS is designed to be modular and initially can be implemented with a more conventional AO relay. The relay replicates the telescope optomechanical feed so that when AM2 is ready and deployed, the AO relay is simply removed from the optical train. 
The initial implementation of MIRAO was chosen to comprise of the following five sub-systems:

- A 1:1 relay with a deformable mirror.

- 3 laser guide star wavefront sensors (LGS WFS).

- A fast, low-order (tip/tilt/focus/astigmatism- TTFA) natural guide star wavefront sensor; this is physically located inside the MIRES cryostat.

- A high-order natural guide star wavefront sensor; this is also inside the MIRES cryostat.

- A deployable calibration unit (also used for MIRES calibrations).

- A real-time controller (RTC).

The implementation with the adaptive secondary (AM2) is the same, except that the AM2 replaces the relay and deformable mirror.

\subsection{Relay}

The basic layout of the relay is shown in Figure 1 below. The relay is accepts the Nasmyth $f / 15$ feed from the TMT telescope and passes a 60 arcsecond diameter field of view to the science instrument(s). The first transmissive element is the dewar window of the spectrograph.

The asterism of off-axis laser guide stars is picked off at the entrance focus of the relay, passed thru a range compensation 'trombone', then passed through the relay. At the exit of the relay, the corrected wavefront is sent to the science instrument and the LGS WFSs are feed with the off-axis beams.

The deformable mirror in the relay is taken to be a 30x30 element stacked actuator mirror with a pitch of 5-7mm. Its stroke and operating requirements are similar to deformable mirrors that have been delivered to a number of facilities (e.g. Starfire Optical Range).

After the relay, natural guide star (NGS) WFSs are implemented in the science instrument dewar to provide an NGS AO option, a Truth NGS WFS for use during LGS observations, and a tip/tilt/focus NGS WFS.

Details of the optical performance of the relay, including the LGS WFS feeds, are given in Liang et al (2006).

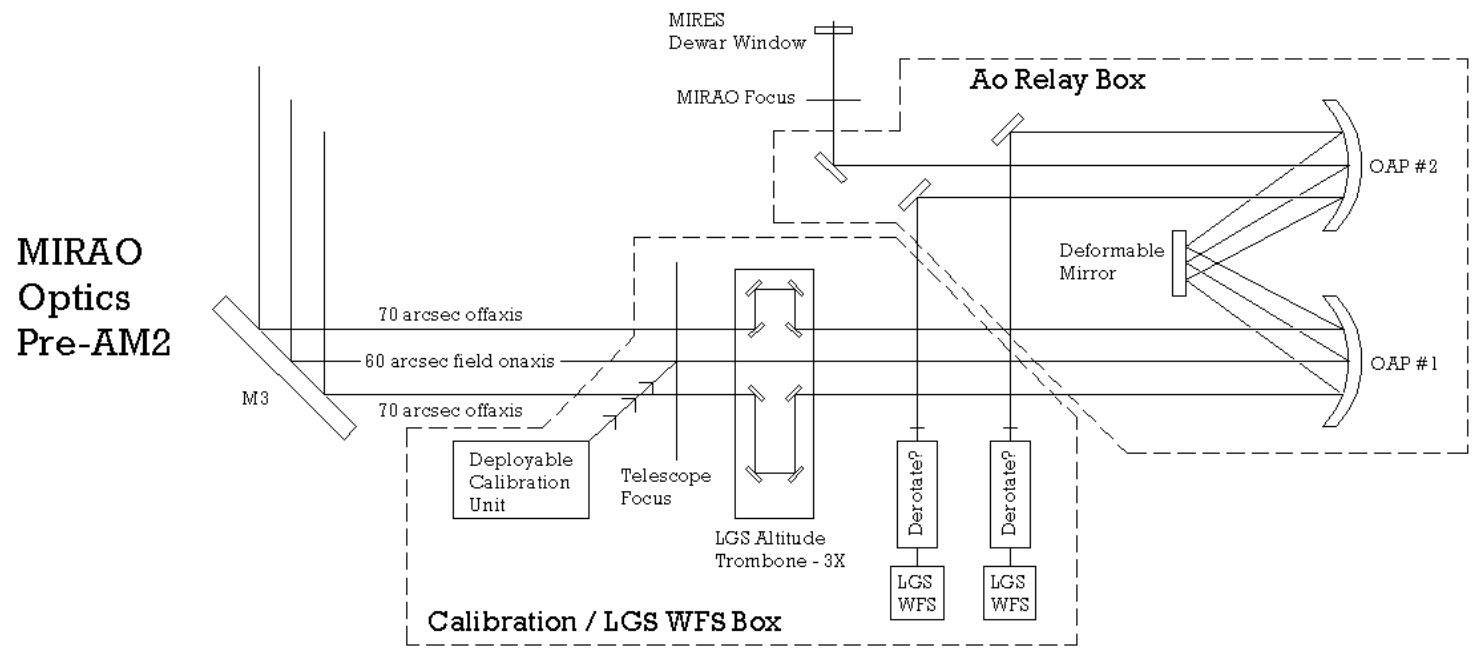

Figure 2. Layout of the MIRAO AO Relay and LGS WFS feeds

\subsection{LGS and LGS WFSs}

The sky coverage and the lack of availability of fast low-noise NIR detectors led us to require the use of laser guide stars. A single sodium laser guide star provides adequate optical performance at the longer wavelengths (10-20 microns) but if placed on-axis would require a transmissive beam splitter. Given the likely availability of multiple laser guide stars from 
the TMT LGS facility, we instead chose to implement multple LGSs off-axis and avoid the transmissive beam splitter. Laser Power requirements are low compared to other TMT AOS requirements but we note that we have made the assumption that there is sufficient power from the facility lasers to produce bright sodium laser guide stars.

The constellation of LGS was chosen to be three off-axis LGS $s$ at a radius of 70-75" from the center of the science field of view. This provides a good correction at the center of the field of view, a large enough separation of the optical beams from the LGSs and from infinity (e.g. The NGSs) to use pickoff mirrors that do not vignet the NGS FOV, provides a sufficiently large NGS field of view for near all-sky coverage, and results in a reasonably sized dewar window. The LGS asterism is kept at a fixed position on the sky by the laser guide star launch telescope so the sets of LGS pickoff mirrors in the relay are stationary. We note that this precludes the operational mode of dithering the LGSs around the science field but since the science FOV is small we do not consider this a limitation.

The LGS WFSs have not been designed in detail. They should not present any major impediments and could even be duplicates of the NFIRAOS LGS WFSs which are initially 60 x 60 subapertures. The over sampling of the wavefront correction may be important for the adaptive secondary implementation. Here, unlike current AM2 systems, the number of controlled AM2 modes is large (e.g. 900) and the registration between the AM2 and the LGS WFS will rotate as the field tracks across the sky if rotation of the LGS WFSs is not included. Simulations at this level of detail are pending.

\subsection{NGS WFSs}

In many cases the science object itself is a good natural guide star (NGS) reference source but given the nature of the science objects the sky coverage is likely significantly better at near infrared wavelengths (1-2 microns).

Three types of natural guide star WFSs are considered though two are envisioned as a single assembly. All of the NGS WFS will reside in the science instrument dewar. After the dewar entrance window the MIR light is split off and sent to the MIR spectrograph/imager. The near infrared light is split into three function: tip/tilt/focus NGS WFS (J-band), NGS AO WFS (H-band), and a HO low-bandwidth "Truth" WFS (H-band). The "Truth" WFS provides a mechanism to feedback changes in the LGS profile or any other calibration offset that may drift during the LGS observations. If a suitable NIR detector is available, the NGS AO WFS and the "Truth" WFS can be combined. The combined WFS is referred to as the HO NGS WFS in the figure below. A trade is still to be done on the precise wavelength split between the respective NGS WFSs but we assume that a single NGS feeds all of the NGS WFSs. Note that the sky coverage calculations were done for a Tip/Tilt/Focus WFS working at J-band and were used to set the NGS WFS patrol field diameter of one arcminute. The NGS WFS feed could be a simple gimbal mirror at a pupil position. Similar cryogenic designs have been implemented in the past of Gemini instruments such as GNIRS and NIRI.

Atmospheric differential refraction will need to be compensated, as the range in wavelength used in the instrument is large. The requirement is to keep the object centered on the MIR spectrograph slit while correcting tip/tilt/focus in the NIR. We expect that this can be accomplished with a simple lookup table but we note that the MIR imager is also a slitviewing camera so any slow drift/flexure can be compensated here.

An acquisition camera working at $\mathrm{K}$-band is also envisioned. This camera provides general acquisition and refraction compensation but also serves as a comparison for registering the MIR spectra with features seen in the NIR (e.g. $\mathrm{Br}$ gamma emission)

\subsection{Optomechanical Design}

Tokunaga et al. (2006), and Liang et al. (2006) present the mechanical layout and optical design of MIRAO/MIRES in detail. Here we simply note that the upgrade from the initial conventional AO relay to use with an adaptive secondary is straightforward. The AO relay and LGS WFSs are mechanically laid out so that when the AO Relay is removed (DM, two off-axis parabolas, and a flat) the change to the optical feed of the LGS WFS is simply an addition of a set of new flats to feed the LGS beams at the entrance of the telescope into the LGS WFS range compensation 'trombones' (Figure 4 below). 


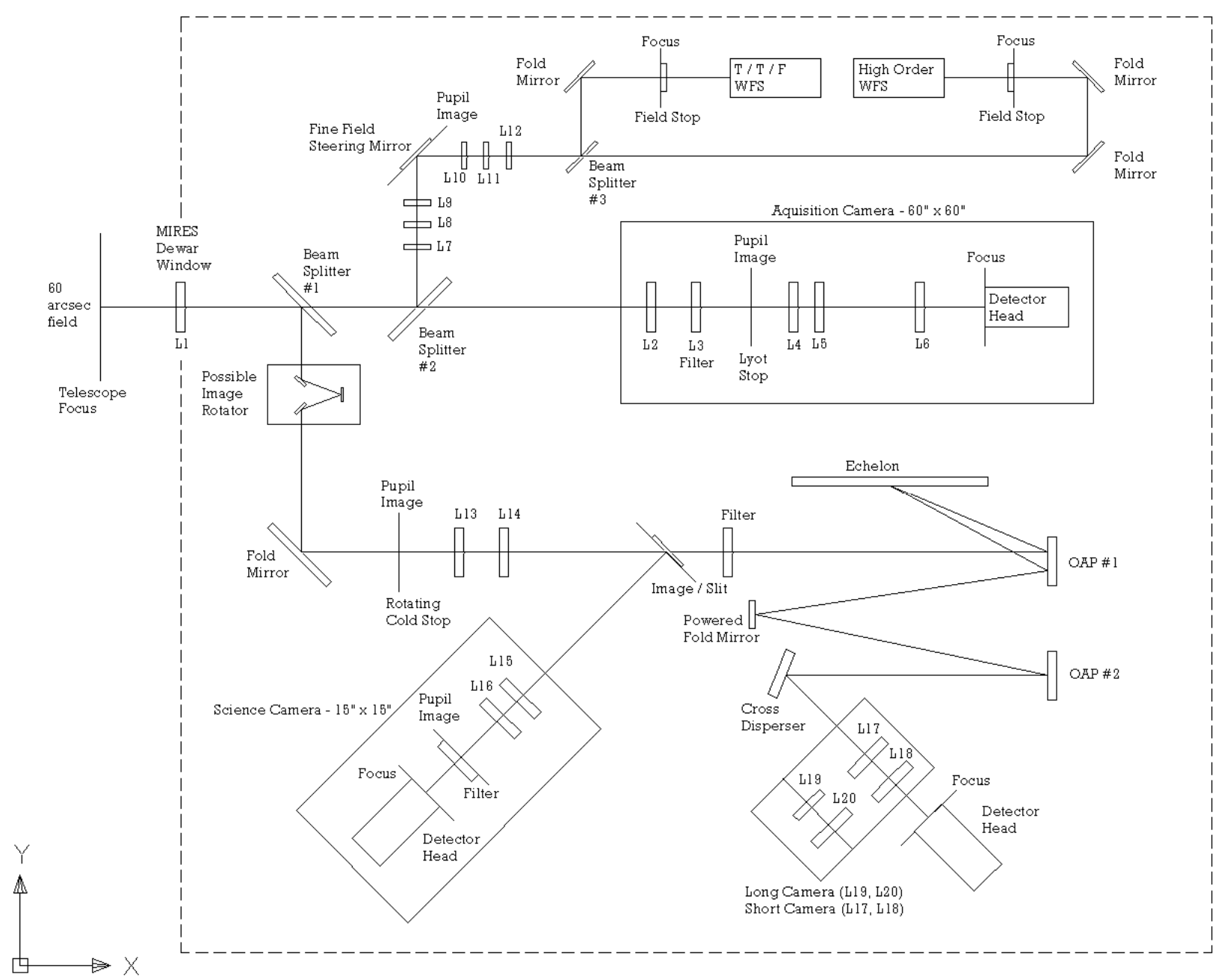

Figure 3 Schematic layout of MIRES and the MIRAO NGS WFSs inside the instrument dewar

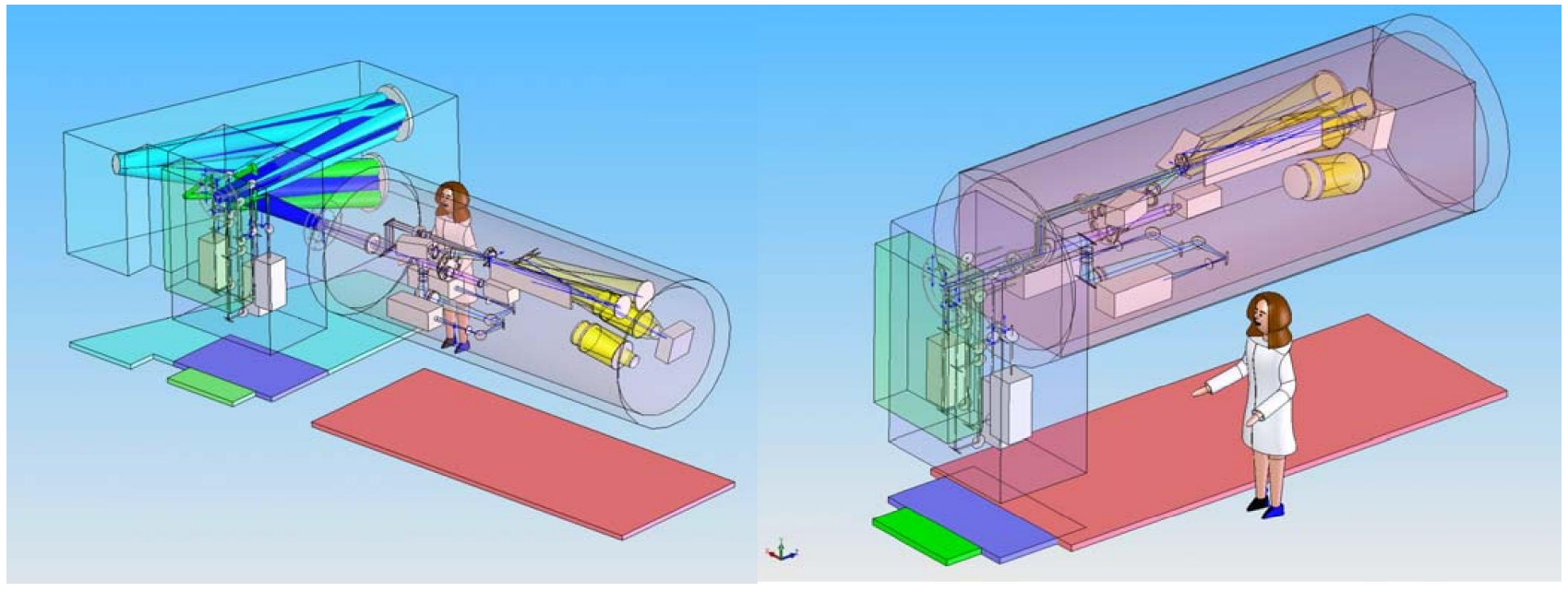

Figure 4 Overall layout and dimensions of the MIRAO/MIRES instruments in the initial AO Relay implementation (left) and in the AM2 configuration (right) 


\section{SUMMARY}

We have completed a feasibility study for an adaptive optics system optimized for use with a mid-infrared echelle spectrograph on TMT. MIRAO is a reasonably low-risk extrapolation of existing adaptive optics systems with the principle risks being the availability of multiple laser guide stars and/or the availability of fast low-noise NIR detectors. The design provides a straightforward approach to achieve the $\mathrm{D}^{4}$ gains possible with a high-resolution mid-infrared spectrograph on TMT.

\section{ACKNOWLEDGMENT}

The authors gratefully acknowledge the support of the TMT partner institutions. They are the Association of Canadian Universities for Research in Astronomy (ACURA), the Association of Universities for Research in Astronomy (AURA), the California Institute of Technology and the University of California. This work was supported, as well, by the Canada Foundation for Innovation, the Gordon and Betty Moore Foundation, the National Optical Astronomy Observatory, which is operated by AURA under cooperative agreement with the National Science Foundation, the Ontario Ministry of Research and Innovation, and the National Research Council of Canada.

\section{REFERENCES}

1. Lloyd-Hart, Michael "Thermal Performance Enhancement of Adaptive Optics by Use of a Deformable Secondary Mirror", PASP, 112, 264, 2000.

2. Elias et al., "Design of the TMT mid-infrared echelle: science drivers and design overview", these proceedings, 2006.

3. $\quad$ Herriot et al., "NFIRAOS", these proceedings, 2006.

4. Liang et al., "Preliminary optical design for the TMT mid-infrared adaptive optics system and echelle spectrograph", these proceedings, 2006.

5. Tokunaga et al., "Design considerations for the high-spectral resolution mid-IR echelle spectrograph on the TMT", these proceeding, 2006. 